\title{
Über die Produktion durchdringender Schauer in verschiedenen Höhenlagen und Materialien, insbesondere in Wasserstoff
}

\author{
Von H. Schultz \\ Aus dem Max-Planck-Institut für Physik, Göttingen \\ (Z. Naturforschg. 9a, 419-431 [1954]; eingegangen am 19. Februar 1954)
}

\begin{abstract}
Zur Entscheidung zwischen den beiden derzeitigen Theorien über die Erzeugung von $\pi$-Mesonenschauern beim Zusammenstoß eines schnellen Nukleons mit einem Atomkern, nämlich der multiplen und der pluralen $\pi$-Mesonenerzeugung, wurde die Materialabhängigkeit der Produktion harter Schauer mit mindestens 3 geladenen Teilchen untersucht.

Die Apparatur zur Messung der Schauerintensität bestand aus einer Anordnung von Bleischichten und Zählrohren, zwischen denen mindestens 8-fach-Koinzidenzen verlangt wurden. Von außen einfallende Schauer konnten durch über der Erzeugersubstanz befindliche Antikoinzidenz-Zählrohre ausgeschaltet werden.

Messungen in verschiedener Höhe über dem Meeresspiegel ergaben einen exponentiellen Intensitätsanstieg mit einer Absorptionslänge $\left(125 \pm 5 \mathrm{~g} / \mathrm{cm}^{2}\right)$, die mit der für die Erzeugung von $\pi$-Mesonenschauern in Photoplatten gefundenen übereinstimmt und die Auslösung der gemessenen Ereignisse durch schnelle Nukleonen beweist. Zur selben Schlußfolgerung führen Absorptionsmessungen mit Bleischichten oberhalb der AntikoinzidenzZählrohre, die als Absorptionslänge die geometrische Kernstoßlänge (160 g/ $\left.\mathrm{cm}^{2}\right)$ ergaben.

An Hand der Ergebnisse von Photoplattenuntersuchungen ließ sich zeigen, daß die mittlere Multiplizität der registrierten Schauer 5-7 betrug, was einer Schauerenergie von $6-12 \mathrm{GeV}$ entsprechen dürfte.

Vergleichsmessungen zwischen Paraffin und Graphit ergaben bei Schichtdicken von $60 \mathrm{~g} / \mathrm{cm}^{2}$ mit einem mittleren Fehler $\pm 2 \%$ dieselbe Koinzidenzzahl pro Minute für beide Substanzen, während beim Ausfall des 15-proz. Wasserstoffanteils im Paraffin ein Unterschied von mindestens $10 \%$ zu erwarten wäre. Da sich zeigen ließ, daß andere Effekte als eine entsprechende Schauerproduktion zur Erklärung des offensichtlich vorhandenen Wasserstoffanteils kaum ausreichen und nach der Theorie der Einzelerzeugung von $\pi$-Mesonen in Wasserstoff Schauer mit mehr als 3 Teilchen nicht auftreten können, wurde darin ein Beweis für die multiple $\pi$-Mesonenerzeugung erblickt.

Zur selben Folgerung führt die Übereinstimmung der Schauererzeugung in Graphit, Aluminium und Eisen, aus der auf einen Erzeugungsquerschnitt je Nukleon geschlossen wurde, der kleiner als der geometrische Stoßquerschnitt ist.
\end{abstract}

\section{Einleitung}

$\mathrm{S}$ eit einigen Jahren weiß man, daß die um 1940 entdeckten ${ }^{1}$ und seitdem oft untersuchten harten (durchdringenden) Schauer in der Höhenstrahlung ${ }^{2}$ aus Garben von schnellen $\pi$-Mesonen und Protonen bestehen, die bei energiereichen Zusammenstößen zwischen der Nukleonenkomponente der Höhenstrahlung und den Atomen der durchsetzten Materie gebildet werden ${ }^{3,4}$. Die Protonen der Schauer dürften fast ausschließlich stark beschleunigte

1 G. Wataghin, M. de Souza Santos u. A. Pompeia, Phys. Rev. 57, 61, 339 [1940]; L. Janossy u. R. Ingleby, Nature, Lond. 145, 511 [1940]; B. Rossi u. V. Regener, Phys. Rev. 58, 837 [1940].

${ }^{2}$ s. Bericht v. K. Sitte, Acta Phys. Austriaca 3, 198 [1950].

3 G. Lattes, G. Occhialini u. C. Powell, Na-
Trümmer der getroffenen Atomkerne sein, während man bezüglich der $\pi$-Mesonen allgemein annimmt, daß sie als eine Art Bremsquanten des die $\mathrm{Nu}$ kleonen begleitenden $\pi$-Mesonenfeldes anzusehen sind. Über den Vorgang der $\pi$-Mesonenerzeugung selbst gehen die Meinungen zur Zeit jedoch noch auseinander.

Nach Heisenberg können bei einem einzigen energiereichen Zusammenstoß zweier Nukleonen gleichzeitig mehrere $\pi$-Mesonen erzeugt werden (multiple Erzeugung von $\pi$-Mesonenschauern, Viel-

ture, Lond. 160, 453 [1947]; R. Brown, U. Camerini, D. Fowler, H. Muirhead, C. Powell u. D. Ritson, ebd. 163, 47. 82 [1949].

${ }^{4}$ U. Camerini, J. Davies, P. Fowler, C. Franzinetti, H. Muirhead, W. Lock, D. Perkins u. G. Yekutieli, Phil. Mag. 42, 1241 [1951]. 
facherzeugung $)^{5}$. Nach Heitler und Janossy ist jedoch ein derartiger Prozeß wegen der hohen Strahlungsdämpfung unwahrscheinlich, so daß beim $\mathrm{Zu}$ sammenstoß zweier Nukleonen fast immer nur ein $\pi$-Meson erzeugt werden kann. $\pi$-Mesonenschauer können nach dieser Auffassung nur in nukleonenreichen Kernen durch eine Folge von sekundären Zusammenstößen entstehen (plurale Schauererzeugung, Einzelerzeugung von $\pi$-Mesonen $)^{6}$.

Experimente über die Schauerentstehung in gröBeren Atomkernen, z. B. Ag, Br (photographische Emulsionen), geben bei dem heutigen Stand der Theorien keine eindeutige Entscheidung für eine der beiden Auffassungen ${ }^{4}$. Dagegen sollte es möglich sein, die Entscheidung durch Experimente mit leichten Atomkernen, insbesondere mit Wasserstoff, herbeizuführen, da dabei größere Schauer nur gemäß der Auffassung von $\mathrm{H}$ e i s e n ber g, nicht aber nach der von Heitler und Janossy, entstehen können.

Bei den nachfolgend beschriebenen Untersuchungen wurde deshalb die Änderung der Schauerproduktion in verschiedenen, unter anderem auch wasserstoffhaltigen Materialien in Abhängigkeit von der Zahl der Schauerteilchen nach der Zählrohrmethode bestimmt (siehe auch die vorläufige Mitteilung ${ }^{7}$ ). Die ursprünglich in Göttingen (160m ü.M.) durchgeführten Messungen wurden später aus Intensitätsgründen in größerer Höhe, im Harz (820 m ü. M.) und auf der Zugspitze (2960 m ü. M.), fortgesetzt. Von anderer Seite veröffentlichte Untersuchungen zum gleichen Thema sollen zusammen mit den eigenen Resultaten in Abschn. IV, C besprochen werden.

\section{Experimentelle Anordnung}

\section{A) Aufbau der Apparatur}

Zur Registrierung der aus der schauererzeugenden Substanz S austretenden harten Schauer diente eine Anordnung von drei Zählrohrlagen (L 1, L 2, L 3) zu je 10 Zählrohren, die so zwischen Bleischichten von $5 \mathrm{~cm}$ Dicke eingebaut und zusammengeschaltet waren, daß die gemessenen Koinzidenzen zwischen den Zählrohren fast nur von Schauern mehrerer durchdringender, geladener Teilchen ausgelöst werden konnten (Abb. 1). Von oben einfallende Luftschauer wurden dabei durch eine über $\mathbf{S}$ befindliche Antikoinzidenz-

${ }^{5}$ W. Heisenberg, Z. Phys. 113, 61 [1949]; 126, 569 [1949]; 133, 65 [1952]; H. Lewis, J. Oppenheimer u. S. Woythusen, Phys. Rev. 73, 127 [1948]; E. Fermi, Progr. Theor. Phys. 5, 570 [1951]; Phys. Rev. 81, 683 [1951].

${ }^{6}$ W. Heitler, Proc. Camb. Phil. Soc. 37, 291 [1941]; lage (La) von 30 Zählrohren ausgeschaltet. Oberhalb davon konnte für Absorptionsversuche mittels eines Kranes noch eine weitere Absorberschicht A angebracht werden.

Alle Zählrohre waren $1 \mathrm{~m}$ lang (effektive Länge $96 \mathrm{~cm}$ ), hatten $3 \mathrm{~cm}$ Durchmesser, einen $0,02 \mathrm{~cm}$ dicken Zähldraht und waren mit einer Mischung von $90 \%$ Argon und 10\% Äthylalkohol bei einem Gesamtdruck von $12 \mathrm{~cm} \mathrm{Hg}$ gefüllt. Mit einer Kathodenspannung von $1250 \mathrm{~V}$, die gemeinsam an die Mäntel aller Rohre einer Lage gelegt wurde, erhielt man Auslöseimpulse von $40-50 \mathrm{~V}$, deren Größe mittels getrennter Vorspannungen an den Zähldrähten für jedes Zählrohr einzeln nachreguliert werden konnte.

Das Plateau der Zählrohre erstreckte sich über einen Bereich von $200-400 \mathrm{~V}$ bei einem relativen Anstieg der Impulszahl von etwa $1 \%$ je $100 \mathrm{~V}$ Spannungsvergrößerung. Unter normalen Betriebsbedingungen lag die Zählspannung etwa $50 \mathrm{~V}$ über dem Plateauanfang. Bei 800 Impulsen pro Minute (im Freien in Göttingen) betrug die Lebensdauer dann etwa $1-1,5$ Jahre.

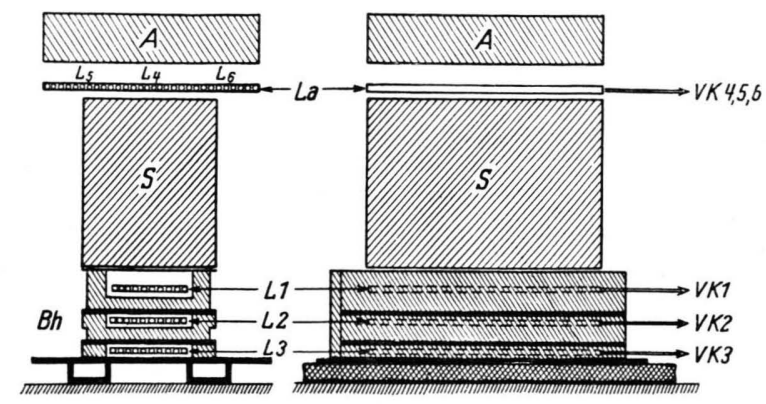

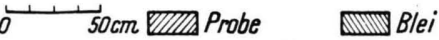

Abb. 1. Versuchsaufbau. In dem Bleihaus Bh befinden sich die drei Zählrohrlagen L 1-L 3, darüber die Substanz S (Blei, Eisen, Kohle oder Paraffin), darüber die Anti-Koinzidenzlage La. A ist eine Bleischicht, mit der die Absorbierbarkeit der schauerauslösenden Strahlung untersucht wird. Kreuzschraffierung bedeutet Eisen.

\section{B) Koinzidenzschaltung}

Die Auswahl der gewünschten Koinzidenzen erfolgt in zwei Stufen. Wie aus dem schematischen Schaltbild (Abb. 2) hervorgeht, werden in sogenannten ,Vorkästen" die gewünschten Koinzidenzen zwischen den 10 Zählrohren einer einzelnen Zählrohrlage ausgesiebt. Die so vorsortierten Koinzidenzen der Lagen werden dann ,Kanalkästen“ zugeleitet, die durch die Art ihrer Anschaltung an sogenannte ,Kanäle“ bestimmen, welche Koinzidenzbedingungen zwischen den Lagen als Ganzes erfüllt sein müssen, damit ein Koinzidenzereignis zur Registrierung kommt.

Phys. Rev. 64, 78 [1943]; Rev. Mod. Phys. 21, 113 [1949]; Helv. Phys. Acta 23, 417 [1950]; L. Janoss y, Phys. Rev. 64, 345 [1943].

7 O. Haxel u. H. Schultz, Z. Naturforschg. 9a, 178 [1954]. 
Auf Grund von Vorversuchen erschien es bei der Anschaltung der 10 Zählrohre an die 5 Koinzidenzröhren eines Vorkastens günstig, immer 2 durch 4 andere Rohre getrennte Zählrohre auf dieselbe Koinzidenzröhre $\mathbf{R}$ zu legen. Eine zwischengeschaltete Verkürzungsschaltung $\mathbf{K}$ bewirkte dabei, daß die Signale an den Koinzidenzröhren $\mathbf{R}$, weitgehend unabhängig von den Eingangsimpulsen, immer die gleiche Form und Länge (etwa $7 \mu$ sec) hatten. Von den am gemeinsamen Anodenwiderstand (,,Rossi“-Widerstand) entstehenden Koinzidenzimpulsen wurden über ,Abschneideröhren" (entweder im Vorkasten oder im Ka- näle angeschaltet, an deren Ausgang die endgültigen Koinzidenzen mittels Endstufen Eund Zählwerken ZW sortiert und gezählt wurden. Die Koinzidenzbedingungen ließen sich somit durch die Zusammenschaltungen der Kanalkästen und die Einstellungen der Abschneideröhren festlegen.

Die Apparatur wurde täglich mit einem Oszillographen und künstlichen Impulsen geprüft und gegebenenfalls nachreguliert. Zur Ausschaltung von Störungen aus dem Netz oder der Umgebung dienten Antikoinzidenzimpulse besonderer Kanalkästen, die durch geeignete Antennen ausgelöst wurden. Die Strom-
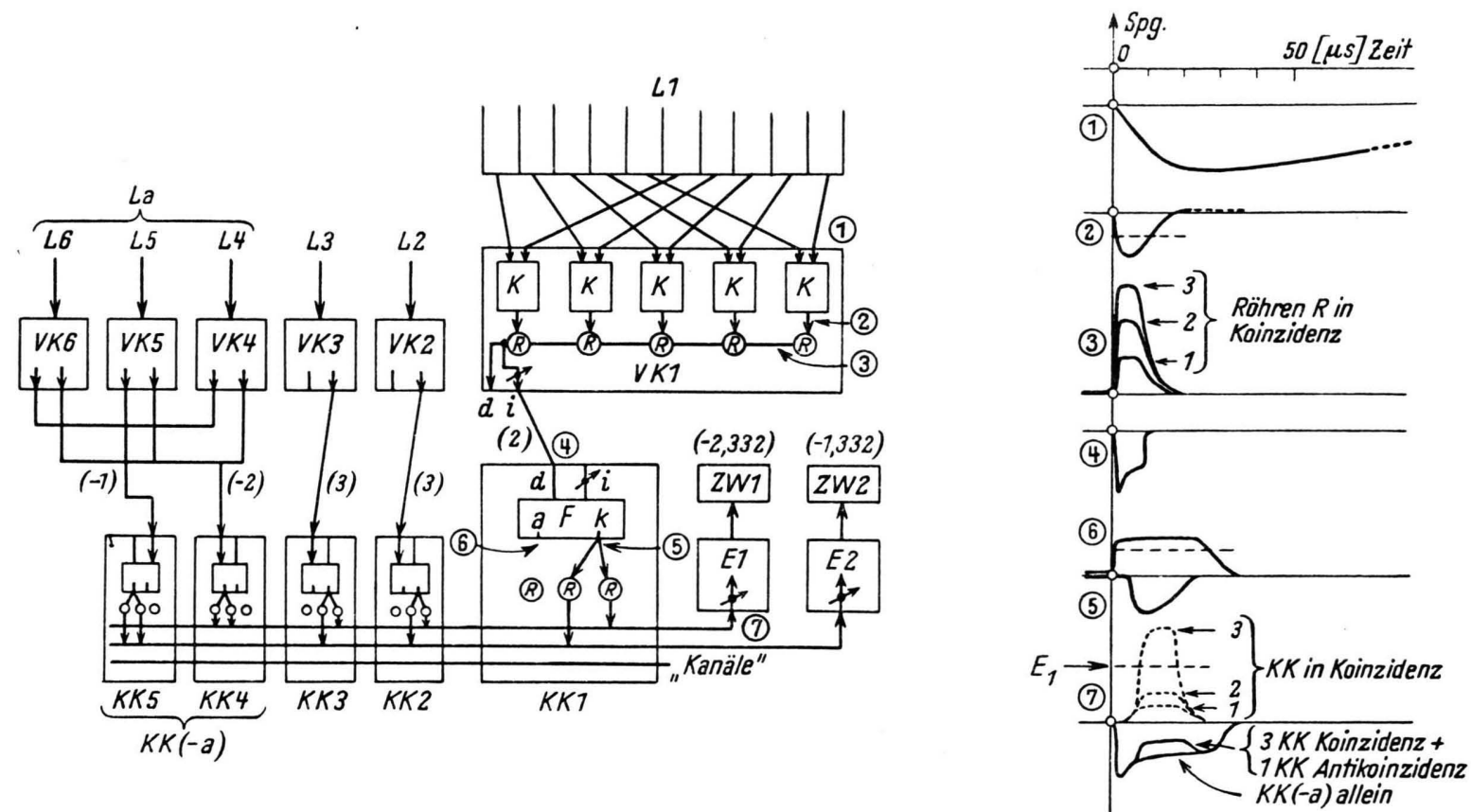

Abb. 2. Blockschema der Koinzidenz-Schaltung. Die Zählrohre einer Lage (L 1-L 6) sind mit abgeschirmten Kabeln mit den Vorkästen (VK $1-$ VK 6) verbunden, wie es für den Fall des VK 1 im Detail dargestellt ist. In den Kanalkästen KK werden die gewünschten Koinzidenz-Kombinationen hergestellt und über die Endstufen E mit den zugehörigen Zählwerken ZW verbunden. Betreffs weiterer Einzelheiten siehe Text Seite 421 . Es bedeuten weiterhin:

K: Verkürz.-Schaltung R: Rossi-Röhren F: Verform.-Elemente

d: direkter Ein- bzw. Ausgang i: indirekter Ein- bzw. Ausgang (über Abschneideröhre) k: Koinzidenz-Impulse a: Antikoinz.-Impulse

nalkasten) nur die genügend großen Signale (entsprechend bestimmten Mindestkoinzidenzen) an den zugehörigen Kanalkasten weitergegeben. Dort wurden sie in den Schaltungen $\mathrm{F}$ so verformt, verzögert und gepolt, daß an den zugehörigen Rossi-Röhren $\mathbf{R}$ der Kanäle Koinzidenz- (bei k) bzw. Antikoinzidenzimpulse (bei a) von 15 bzw. $20 \mu$ sec Länge entstanden, die sich auch bei gegenseitigen Verzögerungen der Eingangsimpulse von bis zu $3 \mu$ sec noch sicher überlappten. Größenunterschiede der Zählrohrimpulse oder zählrohrbedingte Verzögerungen bei ihrer Auslösung können deshalb die Aussortierung der Koinzidenzen nicht beeinflussen. Die Rossi-Röhren der Kanalkästen wurden mit Schaltern in der gewünschten Kombination an die durch alle Kästen hindurchgehenden Ka- versorgung erfolgte anfangs aus der Gleichspannungsbatterie des Instituts, wurde später aber, um die Anlage transportabel zu machen, ganz auf Wechselstrom umgestellt. Ein besonders konstruiertes Regelgerät hielt die Spannung dabei auf $\pm 2 \%$ genau konstant.

\section{C) Wahl der Koinzidenzbedingung}

Aus noch näher zu erläuternden Gründen wurde hauptsächlich bei einer Koinzidenzbedingung gemessen, die zur Registrierung voraussetzte, daß in der untersten Lage (L 3 ) eine Zwei- oder Mehrfach-Koinzidenz und in den beiden darüberliegenden Lagen $(\mathrm{L} \mathbf{2}$, L 1) je eine Drei- oder Mehrfach-Koinzidenz ausgelöst wurde. Bei Auslösung eines Antikoinzidenz-Signals in der Antikoinzidenzlage La wurden derartige Koinzi- 
denzen wieder gelöscht, d. h. das Ereignis wurde trotz ausreichender Treffer in den drei unteren Zählrohrlagen nicht registriert. Die Vorkästen von La waren meistens so eingestellt, daß dazu entweder schon ein Einzelimpuls allein genügte oder aber eine Zwei- oder Mehrfach-Koinzidenz. Durch Benutzung von zwei Kanälen konnten beide Ereignistypen, für die abkürzend $(-1,332)$ und $(-2,332)$ geschrieben sei, gleichzeitig gemessen werden.

Bei dieser Einstellung wurden somit von oben einfallende Schauer ausgeschaltet und nur Ereignisse zugelassen, bei denen entweder gar kein, (-1,332), oder höchstens ein geladenes Teilchen La durchsetzt, $(-2,332)$. Im Falle (-2,332) kann es zwar gelegentlich wegen der Parallelschaltungen von Zählrohren undVorkästen bei La vorkommen, daß La von mehr als einem Teilchen ohne Auslösung einer Antikoinzidenz durchsetzt wird. Diese Fälle sind jedoch so selten, daß sie im allgemeinen genau so wenig berücksichtigt zu werden brauchen, wie die relativ seltenen Treffer in La, die von rückwärts fliegenden Teilchen ausgelöst werden. Die beiden Antikoinzidenzbedingungen bedeuten deshalb praktisch eine Auswahl zwischen geladenen und neutralen schauerauslösenden Teilchen.

Die Differenz der beiden Meßraten, die somit alle Ereignisse umfaßt, bei denen ein und genau ein geladenes Teilchen durch La hindurch einfällt und die geforderte ,Bleihaus-Koinzidenz" auslöst, soll mit $(+1,332)$ bezeichnet werden, trotz der Inkonsequenz, die darin besteht, daß bei $(-1)$ und $(-2)$ jeweils nur eine Mindestbedingung, bei $(+1)$ dagegen eine exakte Bedingung für die Koinzidenzen in La gemeint ist. Die Schreibweise bei anderen Koinzidenzbedingungen dürfte sich nach dem Gesagten von selbst verstehen.

War die Wahl der Antikoinzidenzbedingungen durch die Ausschaltung von Luftschauern bestimmt, so ergab sich die Bedingung (332) aus dem Bestreben, die harten Schauer gegenüber anderen Prozessen möglichst zu bevorzugen. Verlangte man nämlich in den drei unteren Zählrohrlagen jeweils nur Einzelimpulse oder Zweifachkoinzidenzen, dann wurden fast nur Ereignisse gezählt, die mit dem Durchgang einzelner $\mu$-Mesonen verknüpft sind.

Bei (111), (011), (101), (110) werden weitaus überwiegend die schnellen $\mu$-Mesonen allein registriert, wie sich zeigen läßt, wenn man für deren Richtungsverteilung den Ausdruck $A_{n} \cos ^{n} \vartheta \mathrm{d} \varphi \sin \vartheta \mathrm{d} \vartheta(\varphi, \vartheta=$ räumlichePolarkoordinaten) ansetzt und die für verschiedene Lagenkombinationen berechneten Teilchenströme mit den entsprechenden Meßergebnissen vergleicht. In Übereinstimmung mit den Ergebnissen anderer Autoren $^{8}$ findet man als beste Werte $A_{n}=0,54\left(\mathrm{~cm}^{-2} \mathrm{~min}^{-1}\right.$ ster $^{-1}$ ) und $n=3,3$.

Koinzidenzen, bei denen zusätzlich in einer oder auch in mehreren Zählrohrlagen eine Zweifachkoinzidenz verlangt wird, entstehen entweder durch einzelne schrägfliegende $\mu$-Mesonen, die in einer Lage mehrere Zählrohre nacheinander durchsetzen, oder durch

${ }^{8}$ D. Montgomery, Mon.: Cosmic Ray Physics 1949, S. 131 ; W. Kraushaar, Phys. Rev. 76, 1045 [1949]. gleichzeitige Treffer von $\mu$-Mesonen und den von diesen ausgelösten Knock-on-Elektronen. Unter $5 \mathrm{~cm}$ Blei wurde, wie von anderen Autoren auch, eine mittlere Knock-on-Wahrscheinlichkeit 0,06 je $\mu$-Meson gefunden.

Auf diese Weise lassen sich bei der Koinzidenzbedingung (332) jedoch nur noch bis zu $5 \%$ der gefundenen Meßrate erklären. Wie die später beschriebene Höhenabhängigkeit der Intensität zeigt, handelt es sich bei den übrigen Ereignissen zum überwiegenden Teil um die gesuchten harten Schauer. Aus den gleichen Messungen und aus Absorptionsversuchen ergibt sich weiterhin, daß eine wesentliche Erhöhung dieses Anteils auch bei Koinzidenzbedingungen, die über die geforderten 8-fach-Koinzidenzen hinausgehen, nicht zu erzielen ist. Da die Intensität und damit die Meßgenauigkeit dabei andererseits erheblich abnehmen, wurde das Hauptgewicht der Messungen auf die Koinzidenzbedingung (332) gelegt.

Das zeitliche Auflösungsvermögen der Koinzidenzapparatur war so gut, daß die Fehler in den meisten Fällen in der Größenordnung von $1 \%$ lagen und auf besondere Korrekturen verzichtet werden konnte. Die Elimination der falschen Strahlungsanteile an der

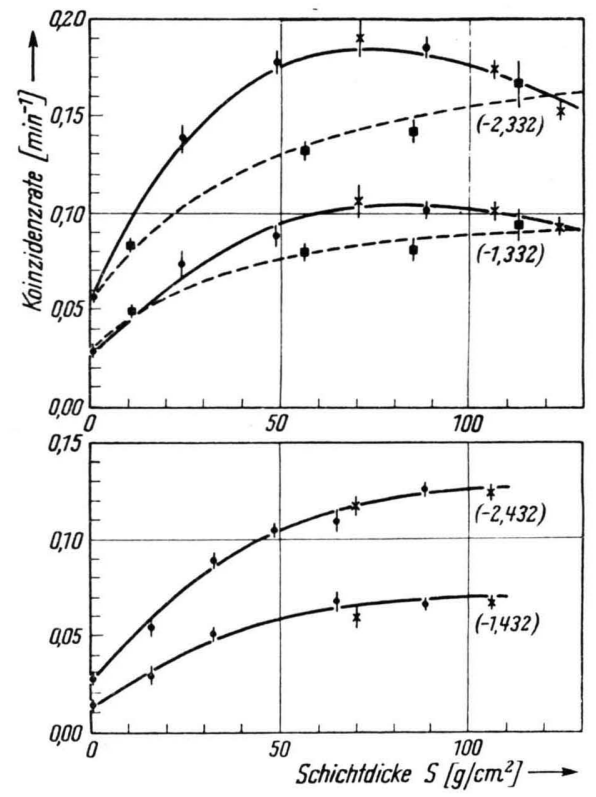

Abb. 3. Zahl der Koinzidenzen (harten Schauer) in Abhängigkeit von der Menge des Materials S. Bei der in der oberen Figur dargestellten Messung wurden in der oberen und mittleren Lage des Bleihauses 3- oder Mehrfach-Koinzidenzen, in der unteren Lage 2- oder Mehrfach-Koinzidenzen verlangt, zusammen also je eine 8 -fach-Koinzidenz. Bei den beiden oberen Kurven wurden durch Antikoinzidenzen alle Ereignisse gelöscht, bei denen in La 2- oder mehr Zählrohre getroffen wurden, bei den beiden unteren Kurven dagegen schon, wenn nur 1- oder mehr Zählrohre ansprachen. Abgekürzte Bezeichnung: (-2,332) und $(-1,332)$. Untere Figur dasselbe für $(-2,432)$ und $(-1,432)$. Grapit; $\times$ Eisen; Blei. 
Meßrate gegenüber der Zahl der harten Schauer wird bei der Besprechung der Meßergebnisse näher behandelt.

\section{Meßergebnisse}

\section{A) Materialabhängigkeit}

Abb. 3 zeigt den Anstieg der Koinzidenzrate, den man beim Aufstapeln von Blei-, Eisen- und Graphitschichten als Erzeugermaterial $S$ über dem Bleihaus mit wachsender Materialdicke (in $\mathrm{g} / \mathrm{cm}^{2}$ gemessen) erhielt. Die schwereren Materialien wurden dabei durch zwischengelegte dünne Holzrahmen auf das Volumen der gleichen Menge Graphit aufgelockert, da besondere Versuche (vgl. z. B. Abb. 5) eine Abhängigkeit der Kurvenform von der

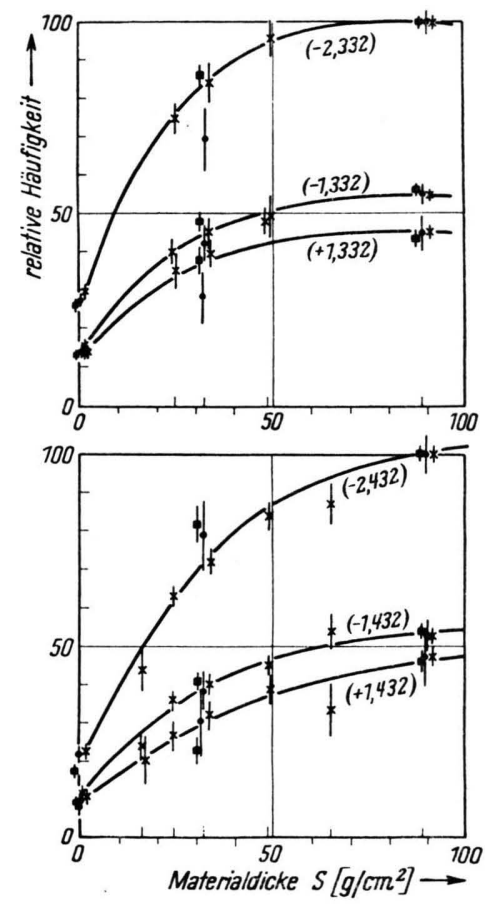

Abb. 4. Harte Schauer aus Graphit in verschiedenen Höhenlagen. Die Messungen mit Graphit in Göttingen ( $\times ; 745 \mathrm{~mm} \mathrm{Hg}, 160 \mathrm{~m}$ ü. M.), im Harz (•;690 mm $\mathrm{Hg}, 820 \mathrm{~m})$ und auf der Zugspitze ( $\mathbf{\square} 535 \mathrm{~mm} \mathrm{Hg}$, $2960 \mathrm{~m}$ ) wurden mit geeigneten Faktoren so normiert, da $B$ die Meßpunkte bei $90 \mathrm{~g} / \mathrm{cm}^{2}$ und Koinzidenz-Bedingung (-2,332), bzw. (-2,432), zusammenfielen.

geometrischen Anordnung des Materials $S$ ergeben hatten. Wie man sieht, kann der Intensitätsanstieg bei Eisen und Graphit innerhalb der Fehlergrenzen durch einen einzigen Kurvenverlauf wiedergegeben werden, zum Unterschied von dem erheblich langsameren Anstieg bei Blei. Hier nicht wiedergegebene Messungen mit Paraffin, Graphit und Aluminium (siehe vorläufige Mitteilung ${ }^{7}$ ), bei denen auf die mittlere Dichte des Paraffins aufgelockert wurde, waren ebenfalls durch eine einzige Kurve darstellbar. Bei gleicher Geometrie erzeugen demnach gleiche Mengen Paraffin, Graphit, Aluminium und Eisen annähernd gleich große Schauerintensitäten, während die Ausbeute bei dem erheblich schwereren Blei, in guter Übereinstimmung mit Messungen von George und Jason ${ }^{9}$, wesentlich kleiner ist. Der Kurvenverlauf bei größeren Materialdicken läßt vermuten, daß bei (332) im Gegensatz zu (432) vorwiegend Schauer aus den unteren Teilen von $S$ gemessen werden.

Graphitmessungen in größeren Höhen, im Harz und auf der Zugspitze, zeigten Intensitätsanstiege auf das 2- bzw. 10-fache. Der Verlauf der Kurven blieb jedoch ungefähr derselbe, wie aus Abb. 4 zu erkennen ist, in der der Ordinatenmaßstab für die verschiedenen Höhen so gewählt wurde, daß die Punkte bei $S=90 \mathrm{~g} / \mathrm{cm}^{2}$ zusammenfallen.

In Abb. 5 sind besonders sorgfältige Vergleichsmessungen an Graphit und Paraffin wiedergegeben, die wegen der größeren Intensität auf der Zugspitze ausgeführt wurden. Bei einer ersten Meßreihe wurde das Paraffin kompakt auf dem Bleihaus aufgebaut (ausgezogene Kurven in Abb. 5) und die zu vergleichende Graphitmenge mit Holzrahmen jeweils soweit aufgelockert, daß sie dasselbe Volumen einnahm (als ,,par-dicht" bezeichnet). Bei einer weiteren Meßreihe wurden alle Paraffin- und Graphitmengen in derselben Weise möglichst gleichmäßig auf das Volumen der maximal verwendeten Paraffinmenge $\left(60 \mathrm{~g} / \mathrm{cm}^{2}\right)$ aufgelockert (gestrichelte Kurven, als ,,vol-konstant" bezeichnet). Aus dem in beiden Fällen verschiedenen Verlauf des Intensitätsanstieges ergibt sich, daß ein eindeutiger Vergleich der Schauerproduktion in verschiedenen Materialien nur bei gleicher geometrischer Anordnung möglich ist.

Da diese Forderung bei den Messungen mit paraffindichter Materialanordnung besonders gut erfüllt werden konnte, sind die zugehörigen Meßergebnisse zum besseren Vergleich in Abb. 6 noch einmal für beide Materialien zusammen eingetragen worden. Wie man sieht, geben Paraffin und Graphit innerhalb der Versuchsfehler, die bei dem Punkt $60 \mathrm{~g} / \mathrm{cm}^{2}$ und Koinzidenzbedingung (332) $\pm 2 \%$ betragen, dieselben Koinzidenzraten. Dieselbe Über-

\footnotetext{
${ }^{9}$ E. George u. A. Jason, Proc. Phys. Soc., Lond. A 63, 1081 [1950].
} 
einstimmung der Intensität dürfte auch bei dünneren Materialschichten bestehen, soweit die größeren Fehlergrenzen eine solche Aussage zulassen. Zwischen $(-1,332)$ und $(-2,332)$ ist, wenigstens bei größeren Materialmengen, ebensowenig ein systematischer Unterschied festzustellen, wie zwischen (332), (432) und (443). Es ist deshalb nicht daran zu zweifeln, daß der Wasserstoffanteil des Paraffins (15\% des Gewichts) an der Koinzidenzerzeugung beteiligt ist, da andernfalls die Paraffinmeßpunkte auf den gestrichelten Kurven in Abb. 6 liegen müßten.

Die eingezeichneten Fehlergrenzen geben den mittleren statistischen Fehler $m$ wieder, der sich gemäß der Formel $m(N / T)=\sqrt{N} / T$ aus der Zahl der Koinzidenzen $N$ und der Meßzeit $T$ errechnet. Eine Nachprüfung mit der Methode der kleinsten

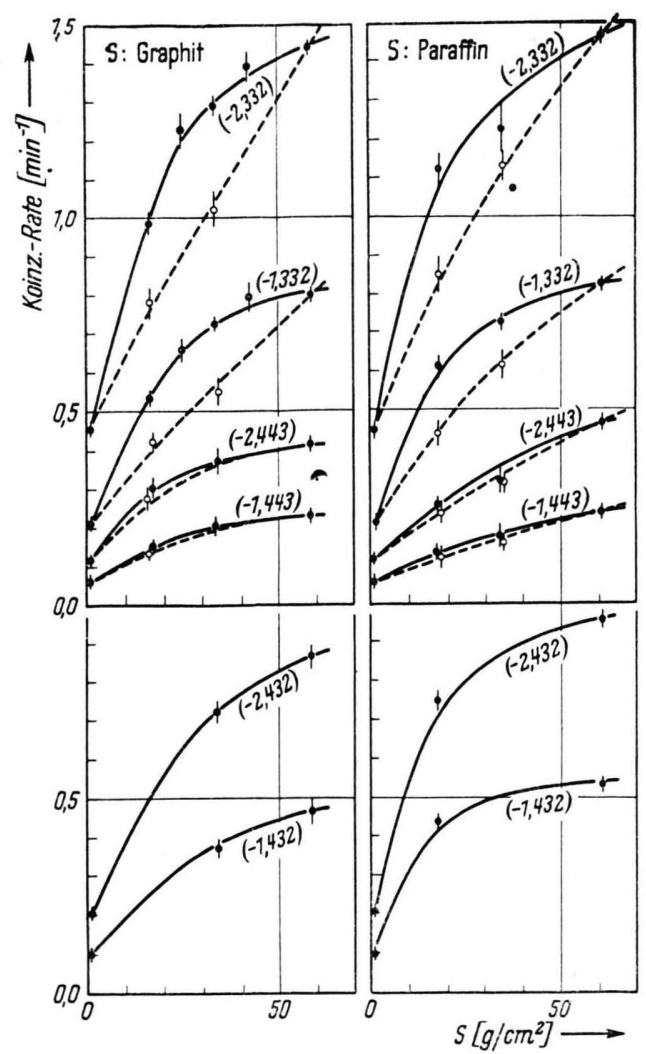

Abb. 5. Koinzidenzauslösung durch harte Schauer aus Graphit, bzw. Paraffin, bei verschiedenen Koinzidenzbedingungen und Materialanordnungen (Zugspitzmessungen). Bei den vollen Punkten (ausgezogene Kurven) war das Paraffin kompakt auf dem Bleihaus aufgestapelt. Graphit wurde so aufgelockert, daß es dasselbe Volumen einnahm wie Paraffin (par-dicht). Bei den Kreisen (gestrichelte Kurven) wurde die jeweilige Materialmenge möglichst gleichmäßig auf das Volumen von $60 \mathrm{~g} / \mathrm{cm}$ Paraffin verteilt (vol-konstant).
Quadrate, die wegen der vielen Einzelmessungen für den Punkt $60 \mathrm{~g} / \mathrm{cm}^{2}$ und (332) möglich war, ergab, daß damit die Fehler richtig wiedergegeben werden.

B) Höhenabhängigkeit und Barometer effekt

Zur Ermittlung der Höhenabhängigkeit der Schauerproduktion wurden in Abb. 7 die in verschiedenen Höhenlagen gefundenen Meßraten mit Graphit in halblogarithmischer Darstellung über dem mittleren Luftdruck in der jeweiligen Höhe aufgetragen (z.Tl. mit verschobenem Nullpunkt der

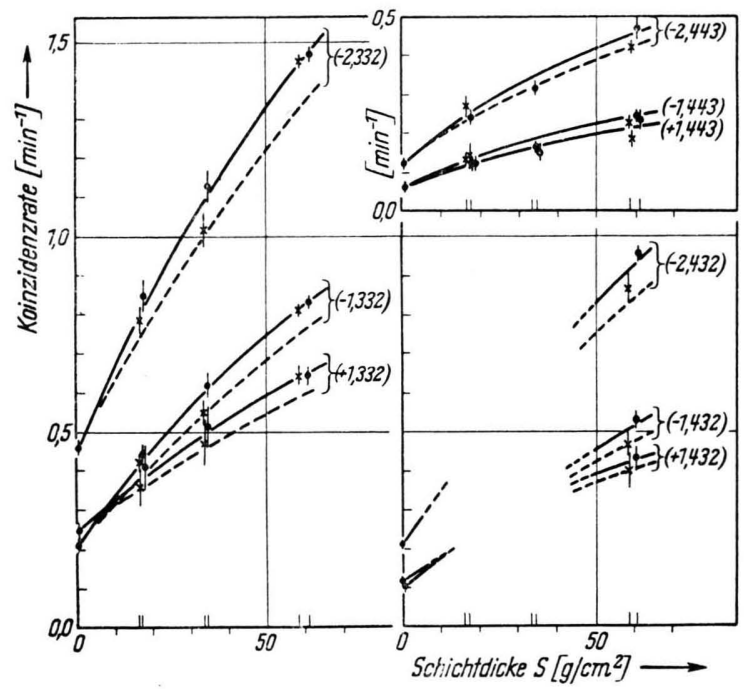

Abb. 6. Vergleich von Paraffin und Graphit (Zugspitzmessungen). • Paraffin, $\times$ Graphit. - Verlauf der gemessenen Koinzidenzzahlen, über beide Substanzen gemittelt; - . - . Koinzidenzzahlen, die man nach den Graphitmessungen für Paraffin zu erwarten hätte, wenn nur der Kohlenstoffanteil desselben zur Schauerproduktion beitrüge. Die Differenz zwischen den beiden Kurven stellt den Anteil des Paraffins dar, den man auf den Wasserstoffgehalt desselben zurückführen muß.

Ordinaten). Wie man sieht, lassen sich die zur gleichen Versuchsanordnung gehörenden Meßpunkte in allen Fällen durch Geraden verbinden. Die mit der Gleichung $I\left(p_{2}\right)=I\left(p_{1}\right) \cdot \exp \left[\left(p_{2}-p_{1}\right) / L\right]$ aus der Steigung der Geraden ermittelten Absorptionslängen $L$ (in $\mathrm{g} / \mathrm{cm}^{2}$ ) sind in der Tab. bei Abb. 7 mit eingetragen worden. Bei $S=35$ und $90 \mathrm{~g} / \mathrm{cm}^{2}$ Graphit ergibt sich somit für $L$ immer etwa derselbe Wert $125 \mathrm{~g} / \mathrm{cm}^{2}$, während für den Nulleffekt (Messungen ohne $S$ ) Werte bis zu $147 \mathrm{~g} / \mathrm{cm}^{2}$ gefunden wurden (besonders bei der Antikoinzidenzbedingung -1$)$. 
Die gleiche Luftdruckabhängigkeit der Schauerzahl erhält man aus dem in Abb. 8 dargestellten Barometereffekt der Messungen. Aus der Neigung der Kurven ergibt sich für Göttingen und die Zugspitze etwa dieselbe Absorptionslänge $L=125 \mathrm{~g} / \mathrm{cm}^{2}$, wie aus der Höhenabhängigkeit, naturgemä $ß$ mit einem erheblich größeren mittleren Fehler, nämlich etwa \pm 20 c $\mathrm{cm}^{2}$.

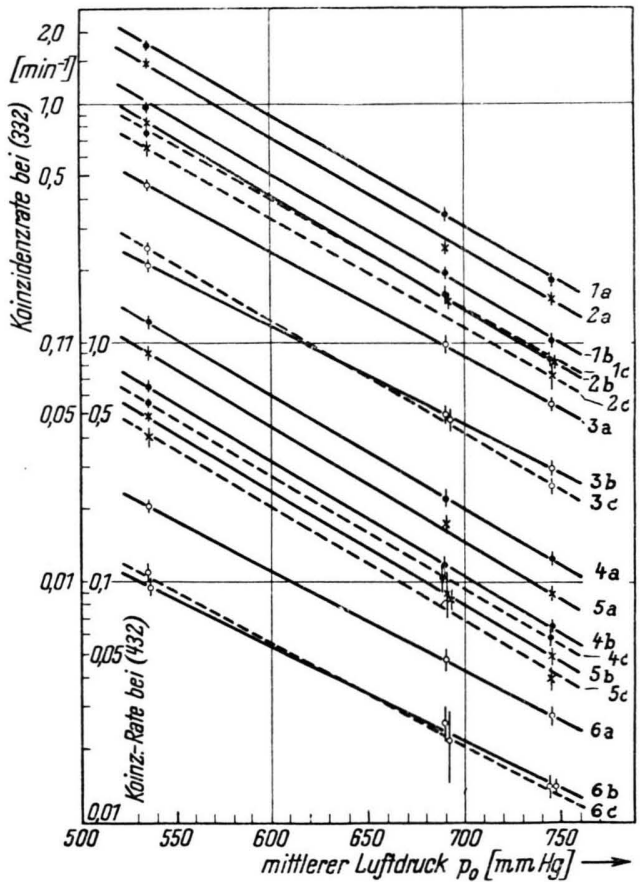

Abb. 7. Höhenabhängigkeit der Schauerproduktion. Aufgetragen sind Messungen an Graphit verschiedener Dicke aus Göttingen (160 m ü. M., $745 \mathrm{mmHg}$ ), Harz $(820 \mathrm{~m}, 690 \mathrm{mmHg})$ und von der Zugspitze $(2960 \mathrm{~m}$, $535 \mathrm{mmHg}$ ). Die Abhängigkeit von der Höhe ist, bis auf den langsameren Anstieg beim Nulleffekt $\left(0 \mathrm{~g} / \mathrm{cm}^{2} \mathrm{C}\right)$, immer dieselbe.

Die Nummern an den Kurven entsprechen folgenden Parametern:

\begin{tabular}{|c|c|c|c|}
\hline Schichtdicke in $\mathrm{g} / \mathrm{cm}^{2} \mathrm{C}$ : & 90 & 35 & 0 \\
\hline Koinz. Bedingung & $\begin{array}{ll} & L \\
\mathrm{Nr} \quad\left(\mathrm{g} / \mathrm{cm}^{2}\right)\end{array}$ & $\begin{array}{ll} & L \\
\mathrm{Nr} \quad\left(\mathrm{g} / \mathrm{cm}^{2}\right)\end{array}$ & $\begin{array}{ll} & L \\
\mathrm{Nr} & \left(\mathrm{g} / \mathrm{cm}^{2}\right)\end{array}$ \\
\hline $\begin{array}{l}(-2,332) \\
(-1,332) \\
(+1,332)\end{array}$ & $\begin{array}{ll}1 \mathrm{a} & 126 \pm 4 \\
1 \mathrm{~b} & 126 \pm 5 \\
1 \mathrm{c} & 126 \pm 6\end{array}$ & $\begin{array}{ll}2 \mathrm{a} & 124 \pm 5 \\
2 \mathrm{~b} & 124 \pm 5 \\
2 \mathrm{c} & 124 \pm 7\end{array}$ & $\begin{array}{ll}3 \mathrm{a} & 135 \pm 3 \\
3 \mathrm{~b} & 142 \pm 6 \\
3 \mathrm{c} & 125 \pm 7\end{array}$ \\
\hline $\begin{array}{l}(-2,432) \\
(-1,432) \\
(+1,432)\end{array}$ & $\begin{array}{ll}4 \mathrm{a} & 124 \pm 5 \\
4 \mathrm{~b} & 122 \pm 5 \\
4 \mathrm{c} & 126 \pm 6\end{array}$ & $\begin{array}{ll}5 \mathrm{a} & 124 \pm 6 \\
5 \mathrm{~b} & 124 \pm 6 \\
5 \mathrm{c} & 124 \pm 8\end{array}$ & $\begin{array}{ll}6 \mathrm{a} & 140 \pm 5 \\
6 \mathrm{~b} & 147 \pm 6 \\
6 \mathrm{c} & 135 \pm 8\end{array}$ \\
\hline
\end{tabular}

\section{C) Absorptionsversuche}

Zur Absorption der primären, schauerauslösenden Strahlung wurden über der Antikoinzidenzlage La verschieden dicke Bleischichten $A$ so angebracht, daß sie möglichst genau den durch La und das Blei- haus definierten Raumwinkel abdeckten. Die dabei gemessene Intensität ist in Abb. 9 in logarithmischem Maßstab über der Absorberdicke $A$ in $\mathrm{g} / \mathrm{cm}^{2}$ aufgetragen.

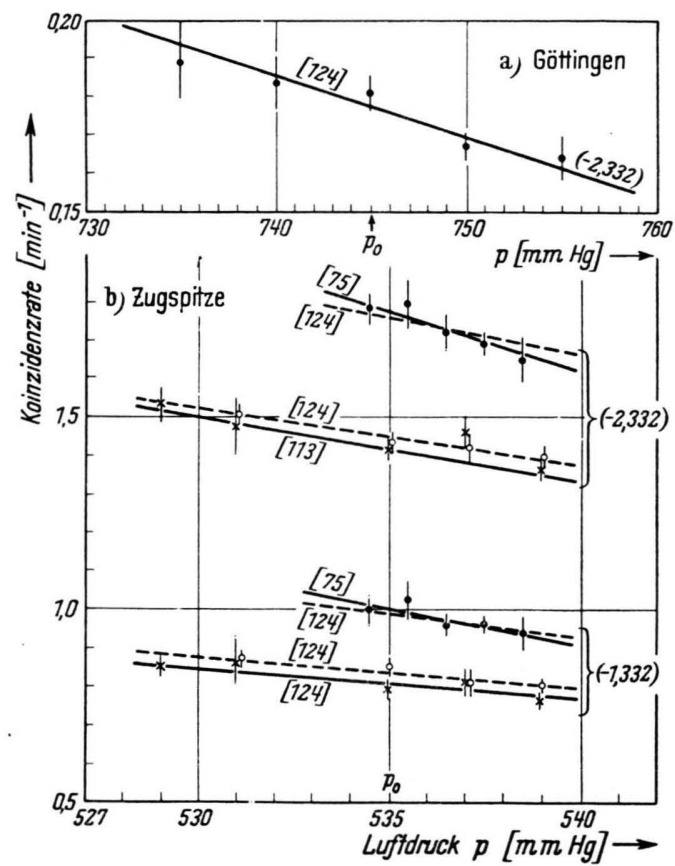

Abb. 8. Barometereffekt. a) Messung auf Seehöhe (Göttingen) mit $90 \mathrm{~g} / \mathrm{cm}^{2}$ Graphit (kompakt) bei Koinzidenz-Bedingung (-2,332); b) Messung in $2960 \mathrm{~m}$ Höhe (Zugspitze); • mit $90 \mathrm{~g} / \mathrm{cm}^{2}$ Graphit (kompakt), $\times$ mit $60 \mathrm{~g} / \mathrm{cm}^{2}$ Graphit (auf Paraff.-Dichte aufgelockert), $\bigcirc$ mit $60 \mathrm{~g} / \mathrm{cm}^{2}$ Paraffin (kompakt).

Die Zahlen in [ ] sind die zugehörigen Werte von $L$ in $\mathrm{g} / \mathrm{cm}^{2}$ gemessen.

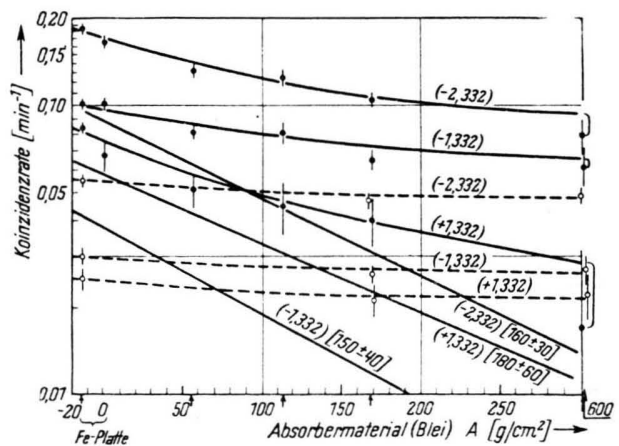

Abb. 9. Absorption der schauerauslösenden Komponente in Blei. Auf der Ordinate ist die Schauerrate (in logarithmischer Skala), auf der Abszisse die Dicke des Blei-Absorbers A (in $\mathrm{g} / \mathrm{cm}^{2}$ ) aufgetragen. - - Messungen bei den Koinzidenz-Bedingungen (-2,332), (-1,332), (+1,332) bei $S=90 \mathrm{~g} / \mathrm{cm}^{2}$ Graphit; - - $\bigcirc--$ entsprechender Verlauf für den Fall $S=0$, d. h. ohne schauererzeugendes Material. Wegen der Bedeutung der schrägen Geraden siehe Seite 426. (Die Zahlen in [ ] sind die zugehörigen Absorptionslängen $L$, in $\mathrm{g} / \mathrm{cm}^{2}$.) 
Der langsam flacher werdendeVerlauf der Kurven legt die Vermutung nahe, daß sich die Zusammensetzung der schauerauslösenden Strahlung mit wachsender Absorberdicke ändert. Zur Untersuchung der Frage, wie weit es sich dabei um zwei verschieden stark absorbierbare Anteile, z. B. Nukleonen und $\mu$-Mesonen, oder um nicht absorbierte, seitlich von La einfallende Primärstrahlen handelt, wurde versucht, durch sehr dicke Absorberschichten jeweils einen der beiden Raumwinkel möglichst gut abzuschirmen.

Dazu wurde die Apparatur als Ganzes so unter eine Luke in der Zwischendecke des Göttinger Labors aufgestellt, daß der seitlich von La einfallende Teilchenstrom durch einen ,WWall“" aus Basaltsteinen abgedeckt werden konnte. Anschließend wurde dieselbe Basaltmenge als pilzförmiger ,Block“ über der Luke, d. h. über La, aufgebaut. Die mittlere Dicke der Absorberschichten betrug im ersten Fall 3, im zweiten Fall 5 geometrische Kernstoßlängen (180 bzw. $320 \mathrm{~g} / \mathrm{cm}^{2} \mathrm{Ba}-$ salt), so daß Nukleonen nur zu einem verschwindenden Teil ohne Stoß in der betreffenden Richtung einfallen konnten. Bei der Messung mit ,Block“ kommt das praktisch der Ausschaltung der vertikalen NukleonenKomponente gleich, da fast alle Stöße im ,Block“ entweder zur Absorption der Primärteilchen oder zur Auslösung von Antikoinzidenzen durch die dabei erzeugten Schauer führen. Der Einfluß des auf den Lukenrand aufgebauten ,Walls " ist demgegenüber erheblich schwerer zu beurteilen, da in diesem Fall auch damit gerechnet werden muß, daß im ,Wall" erzeugte Schauer die erforderliche Bleihauskoinzidenz auslösen können, ohne durch eine Antikoinzidenz gelöscht zu werden.

\begin{tabular}{|c|c|c|c|c|}
\hline \multicolumn{2}{|c|}{$\begin{array}{c}\text { Aufbau des Absorbermaterials } \\
\text { Luke }\end{array}$} & $\begin{array}{c}\text { Erzeuger- } \\
\text { Material } \\
S \\
\end{array}$ & \multicolumn{2}{|c|}{$\begin{array}{l}\text { Koinzidenzzahl } \cdot 10^{3} \text { / } \\
\text { Minute bei } \\
\text { Koinzidenzbedingung: } \\
(-1,332) \mid(+1,332)\end{array}$} \\
\hline $\begin{array}{l}\text { a leer }(0,15) \\
\text { b leer }(0,15) \\
\text { c Block }(5,0) \\
\text { d leer }(0,15) \\
\text { e } 112 \mathrm{~g} / \mathrm{cm}^{2} \mathrm{~Pb}(1,1)\end{array}$ & $\begin{array}{lr}\text { leer } & (0,4) \\
\text { Wall } & (3,0) \\
\text { leer } & (0,4) \\
- & - \\
- & - \\
- & -\end{array}$ & 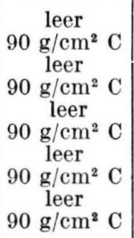 & $\begin{aligned} 33 & \pm 5 \\
105 & \pm 7 \\
31 & \pm 4 \\
99 & \pm 5 \\
35 & \pm 4 \\
69 & \pm 6 \\
30 & \pm 2 \\
101 & \pm 2 \\
26 & \pm 2 \\
65 & \pm 5\end{aligned}$ & $\begin{array}{l}27 \pm 8 \\
91 \pm 12 \\
42 \pm 8 \\
75 \pm 9 \\
25 \pm 7 \\
30 \pm 9 \\
25 \pm 2 \\
84 \pm 2 \\
21 \pm 3 \\
40 \pm 8\end{array}$ \\
\hline
\end{tabular}

Die Messungen a, b, c wurden unter der Luke durchgeführt, die Messungen d, e sind Vergleichsmessungen mit dem normalen Aufbau im Laboratorium. Die eingeklammerten Zahlen geben die Absorberdicken in geometrischen freien Weglängen gemessen an, die sich außer Luft über dem betreffenden Raumwinkel befanden.

Tab.1. Absorptionsmessungen mit der unter der Luke des Göttinger Laboratoriums aufgestellten Apparatur.

Die Zusammenstellung der Meßergebnisse in Tab. 1 ergibt für die Messungen ohne „Block“ oder „Wall“ nur unwesentlich höhere Koinzidenzraten als beim normalen Aufbau der Apparatur im Labor, so daß die Ergebnisse mit dem ,,Block" über La ohne weiteres als Meßpunkte bei besonders dicken
Absorberschichten $A$ in Abb. 9 angesehen werden können. Für die entsprechende Bleischicht ergibt sich etwa $600 \mathrm{~g} / \mathrm{cm}^{2}$, wenn bei der Umrechnung von gleichbleibender Dicke in geometrischen Kernstoßlängen ausgegangen wird. Berücksichtigt man dabei, daß nur etwa $90 \%$ der in La einfallenden Strahlung durch den Block abgedeckt werden, dann ergibt sich in Abb. 9 bei großen Absorberdicken ein so flacher Kurvenverlauf, daß wesentliche Intensitätsänderungen auch bei erheblich größeren Absorbermengen nicht mehr zu erwarten sind. Nach Abzug dieser Restintensitäten von den Meßwerten bei kleinen Absorberdicken erhält man in Abb. 9 annähernd gerade Linien, aus deren Steigung für die Absorptionslängen $L$ in Blei bei $(-1,332) 150 \pm 40$ $\mathrm{g} / \mathrm{cm}^{2}$ bzw. bei $(+1,332) \quad 180 \pm 60 \mathrm{gc} / \mathrm{m}^{2}$ folgt.

\section{Diskussion}

\section{A) Art der schauererzeugenden Strahlen}

Die Höhenabhängigkeit der Schauerproduktion und die Absorptionsversuche zeigen, daß die Auslösung der in der Apparatur registrierten Schauer zum weitaus größten Teil durch schnelle Nukleonen der Höhenstrahlung erfolgt.

Das ergibt sich im ersteren Falle sofort, wenn man die hier beobachtete Absorptionslänge in Luft, $125 \pm 5 \mathrm{~g} / \mathrm{cm}^{2}$, mit der für die Erzeugung entsprechender Schauer in Photoplatten vergleicht, die nach neueren Angaben etwa $130 \mathrm{~g} / \mathrm{cm}^{2}$ beträgt ${ }^{10}$, während ältere Werte zwischen 120 und $150 \mathrm{~g} / \mathrm{cm}^{2}$ lagen. Wegen dieser ausgezeichneten Übereinstimmung ist kaum ernsthaft daran zu zweifeln, daß die hier untersuchten Schauer ebenfalls von der Nukleonenkomponente ausgelöst werden, zumindest bei den Messungen mit $S=30$ bis $90 \mathrm{~g} / \mathrm{cm}^{2}$ Graphit.

Beim Nulleffekt, d. h. bei den Messungen ohne Material $S$, scheint daneben, wie der geringere Intensitätsanstieg mit der Höhe vermuten läßt, noch eine zweite Strahlungsart, vor allem bei der Antikoinzidenzbedingung $(-1)$, merklich beteiligt $\mathrm{zu}$ sein. Bedenkt man, daß schräg einfallende, geladene Teilchen (bei -1) in einer Lage leichter zwei Zählrohre gleichzeitig treffen und dabei eine Koinzidenz auslösen können als die steiler einfallenden $($ bei +1$)$, dann erkennt man, daß hierfür wohl nur $\mu$-Mesonen in Begleitung genügend vieler Knock-on-Elektronen in Frage kommen. Bei einem Intensitätsanstieg zwischen Göttingen und der Zugspitze auf das höch-

${ }^{10}$ M. Teucher, Z. Naturforschg. 7a, 61 [1952]. 
stens 1,7-fache ${ }^{11}$ beträgt der mit der gemessenen Höhenabhängigkeit bei $(-1,332)$ verträgliche $\mu$ Mesonenanteil am Nulleffekt $35 \pm 15 \%$, während der Anteil bei $(+1,332)$ sowie bei allen Messungen mit $S=30$ bis $90 \mathrm{~g} / \mathrm{cm}^{2}$ Graphit nur etwa $5 \pm 10 \%$ ausmachen dürfte. Wegen des erheblich stärkeren Intensitätsanstieges der Nukleonenkomponente mit der Höhe ist deshalb der $\mu$-Mesonenanteil auf der Zugspitze sicherlich zu vernachlässigen.

Die verschiedenen, meist größeren Absorptionslängen, die von anderen Autoren mit Zählrohranlagen aus der Höhenabhängigkeit ermittelt wurden, sind wahrscheinlich ebenso wie hier beim Nulleffekt auf verschieden große $\mu$-Mesonenanteile zurückzuführen. Mit ähnlichen Anordnungen erhielten neuerdings übrigens auch Ticho ${ }^{12}$ und Rollos on ${ }^{13}$ für die entsprechenden Schauer Absorptionslängen, die mit den hier gefundenen praktisch übereinstimmen.

Ein weiterer Beweis für die Schauerauslösung durch schnelle Nukleonen kann darin erblickt werden, daß bei den Absorptionsmessungen mit $S=90$ $\mathrm{g} / \mathrm{cm}^{2}$ Graphit (Abb. 9) nach Abzug der mit ,,Block“ übrig bleibenden Restintensitäten von den Werten bei kleineren Absorberdicken annähernd exponentielle Absorptionskurven erhalten werden, deren Absorptionslängen überraschend gut zur geometrischen Kernstoßlänge in Blei $\left(160 \mathrm{~g} / \mathrm{cm}^{2}\right)$ passen. Es liegt deshalb die Vermutung nahe, daß die hier beobachtete Absorption durch Kerntreffer mit dem geometrischen Kernquerschnitt erfolgt, und daß es sich folglich um schnelle Nukleonen handelt. Das gilt besondersfürdieKoinzidenzbedingung (-1,332), da außer Neutronen keine neutrale Strahlung von genügender Häufigkeit und Absorbierbarkeit bekannt ist. Die nach Abzug der Restintensitäten übrig bleibende Absorptionskurve dürfte deshalb in diesem Falle direkt die Absorption der Neutronen durch den Absorber $A$ wiedergeben.

Bei $(+1,332)$ gilt dasselbe nicht so ohne weiteres, da hier mit einem von oben einfallenden $\mu$-Mesonenanteil gerechnet werden muß, der durch den ,,Block “ nur zu etwa $30 \%$ absorbiert würde. Angenommen, die ganze mit ,Block“ verbleibende Restkoinzidenzrate würde nur von solchen $\mu$-Mesonen erzeugt, dann hätte man bei den kleineren Absorberdicken einen mit abnehmender Dicke bis auf das 1,3-fache linear ansteigenden Anteil statt der konstanten Rest-

11 B. Rossi, Rev. Mod. Phys. 20, 537 [1948].

${ }^{12}$ K. Ticho, Phys. Rev. 88, 237 [1952]. intensität abzuziehen, um die Absorption der Protonen zu erhalten. Wegen der seitlich an La vorbei einfallenden Nukleonen und $\mu$-Mesonen wird der $\mu$-Mesonenanteil in Wirklichkeit jedoch erheblich kleiner sein. Eine sorgfältige Abschätzung dieser Anteile auf Grund der bekannten Richtungsverteilungen ergab für $S=90 \mathrm{~g} / \mathrm{cm}^{2}$ Graphit ohne Absorber etwa die folgende Aufteilung auf die einzelnen Komponenten: Bei $(+1,332): 20 \% \mu$-Mesonen, $15 \%$ seitliche Nukleonen, $65 \%$ durch La einfallende Protonen; bei $(-1,332)$ : $15 \% \mu$-Mesonen, $45 \%$ seitliche Nukleonen, $40 \%$ durch La einfallende Neutronen. Bei den $\mu$-Mesonen handelt es sich um Maximalwerte, da außer schauerauslösenden Einzelteilchen auch noch direkte seitliche Schauer zur Restintensität beitragen können. Für die Messungen ohne schauererzeugendes Material $S$, d. h. für den Nulleffekt, ist eine entsprechende Abschätzung wegen der geringen statistischen Genauigkeit leider nicht durchführbar, wenn sich auch zeigen läßt, daß kein ernsthafter Gegensatz zu den Resultaten der Höhenabhängigkeit besteht.

Unter Berücksichtigung des veränderlichen $\mu$ Mesonenanteils erhält man für den Nukleonenanteil der (+1,332)-Absorptionskurve bei $90 \mathrm{~g} / \mathrm{cm}^{2}$ Graphit in Abb. 9 eine etwas steilere Gerade als nach Abzug der konstanten Restintensität allein. Die entsprechend kleinere Absorptionslänge paßt dann innerhalb der Meßgenauigkeit ebensogut zur geometrischen Kernstoßlänge in Blei, wie die bei $(-1,332)$ gefundene.

Man wird daraus schließen dürfen, daß in beiden Fällen die schauerauslösenden Teilchen Nukleonen sind; darüber hinaus aber auch, daß die registrierten Schauer im Mittel mehr als 3 geladene Teilchen enthalten. Die gute Übereinstimmung zwischen Absorptions- und Stoßlänge läßt nämlich vermuten, daß die Absorption hier vorwiegend dadurch erfolgt, daß bei den Stößen in $A$ Schauer entstehen, welche in La eine Antikoinzidenz auszulösen vermögen, da die Stöße andernfalls oft nur zu Energieverlusten, nicht aber zum totalen Ausfall der Teilchen führen würden. Wegen der geometrischen Voraussetzungen für die Auslösung von (-2)-Antikoinzidenzen darf man aber annehmen, daß dazu mindestens 3 geladene Teilchen nach einem Stoß in $A$ erforderlich sind, mit andern Worten, daß die im Bleihaus registrierten Teilchen bei fast jedem

13 G. Rolloson, Phys. Rev. 87, 71 [1952]. 
Kernstoß in Blei Schauer mit 3 oder mehr harten Sekundärteilchen erzeugen.

Aus der Abschätzung der Strahlungsanteile an der Koinzidenzerzeugung bei $90 \mathrm{~g} / \mathrm{cm}^{2}$ geht weiterhin hervor, daß etwa 1,6-mal mehr von oben einfallende Protonen als Neutronen registriert werden. Für das richtige Zahlenverhältnis zwischen den beiden Teilchenarten innerhalb des beobachteten Energieintervalls muß jedoch berücksichtigt werden, daß die von $S$ ausgehenden Rückwärtstreffer der Schauer das Meßratenverhältnis um etwa 10\% gegenüber dem Verhältnis der entsprechenden Teilchenströme vergrößern, und daß die von Protonen erzeugten Schauer im Mittel immer ein geladenes Teilchen mehr enthalten, als die von energiegleichen Neutronen ausgelösten, was zu einer Zunahme der Zählwahrscheinlichkeit um 10-15\% führt (s. nächster Abschnitt). Das wirkliche Proton/Neutron-Verhältnis in dem hier beobachteten Energiebereich $3-20 \mathrm{GeV}$ (s. nächster Abschnitt) dürfte deshalb $1,2 \pm 2$ betragen, in Übereinstimmung mit den Resultaten ähnlicher Zählrohrmessungen ${ }^{14,15}$, bei denen $1-1,5$ gefunden wurde.

Die bisherigen Schlüsse werden endlich noch bestätigt, wenn man das Zahlenverhältnis zwischen den seitlich von La und den durch La einfallenden Nukleonen, das sich nach den Abschätzungen der Strahlungsanteile ergibt, mit dem auf Grund einer $\cos ^{n}$-Verteilung berechneten vergleicht. Die beste Übereinstimmung besteht dann bei $n=6-8$, Werten für $n$, die auch von anderen Autoren gefunden wurden ${ }^{16}$.

\section{B) Art der gemessenen Schauer}

Für den Vergleich der Meßergebnisse bei verschiedenen Materialien und Koinzidenzbedingungen muß neben den Eigenschaften der Primärteilchen der Schauer auch deren Zusammensetzung selbst einigermaßen bekannt sein, da sonst die Abhängigkeit der Meßrate von Unterschieden der Schauerstruktur nicht abgeschätzt werden kann.

So würden beispielsweise alle Schauer, die außer den geladenen auch neutrale $\pi$-Mesonen enthalten, wegen der großen Elektronenkaskaden, die bei deren Zerfall in zwei $\gamma$-Quanten entstehen, erheb-

${ }^{14}$ W. Brown u. A. Mc Kay, Phys. Rev. 77, 343 [1950].

${ }^{15} \mathrm{~W}$. Walker, N. Duller u. J. Sorrels, Phys. Rev. 86, 865 [1952].

${ }_{16}$ M. Mylroi u. J. Wilson, Proc. Phys. Soc., Lond. A 64, 404[1951]. F. Froehlich, E. Harth u. K. Sitte, lich besser registriert werden als gleiche Schauer mit ausschließlich geladenen $\pi$-Mesonen, vorausgesetzt, daß die Kaskaden mehrere Zählrohrlagen auf einmal durchsetzen können. Die Mindestenergie dafür beträgt nach neueren Rechnungen 0,3 bzw. $1 \mathrm{GeV}$ (für 5 bzw. $10 \mathrm{~cm}$ Blei) ${ }^{17}$, während die mittlere Energie der $\pi$-Mesonen nach dem Energiespektrum der Schauerteilchen von Camerini et al.4,18 höchstens 0,6 GeV betragen dürfte. Wegen der Aufteilung der $\pi$-Mesonenenergie auf zwei $\gamma$-Quanten ist eine ernsthafte Verfälschung der Zahl der alle drei Zählrohrlagen durchsetzenden Schauerteilchen durch solche Elektronenkaskaden also nicht zu befürchten. Aber auch bei den Koinzidenzen zwischen nur zwei Zählrohrlagen dürften Störungen dieser Art wegen der Richtungsverteilung der $\pi$-Mesonen und der Bedingung, daß mindestens ein Elektronenpaar oberhalb der oberen Zählrohrlage L 1 erzeugt wird, nicht allzusehr ins Gewicht fallen. Auf die besonderen Verhältnisse beim Wasserstoff wird weiter unten näher eingegangen.

Im Prinzip genügt zur Auslösung einer (332)Koinzidenz schon ein Schauer mit nur 3 geladenen Teilchen; im allgemeinen werden dazu wegen der komplizierten geometrischen Koinzidenzbedingungen jedoch erheblich mehr Teilchen benötigt. Die besser registrierten, größeren Schauer kommen andererseits so viel seltener vor, daß es eine optimale Teilchenzahl der Schauer geben muß, oberhalb der die geringere Erzeugung, unterhalb der die kleinere Zählwahrscheinlichkeit überwiegt.

Diese beiden, die gemessene Multiplizitätsverteilung bestimmenden Effekte hängen jedoch selbst von so vielen Parametern ab, daß ihre Berechnung auf Grund der Meßergebnisse allein unmöglich sein dürfte. Es wurde deshalb der umgekehrte Weg eingeschlagen und die Verteilung zunächst mit den aus Photoplattenmessungen bekannten Daten über harte Schauer berechnet und das Ergebnis mit den Messungen verglichen.

Von den Absorptionseffekten des Materials $S$ gegenüber den Primär- und Sekundärteilchen wurde dabei abgesehen und angenommen, daß die Schauer überall mit gleicher Häufigkeit entstehen und daß oberhalb des Bleihauses keine Ablenkungen oder

Phys. Rev. 87, 504 [1952]; W. Walker, ebd. 77, 686 [1950].

17 K. Ott, s. Anhang 4 a in W. Heisenberg, Kosmische Strahlung, 1953 , S. 523.

18 U. Camerini, P. Fowler, W. Lock u. H. Muirhead, Phil. Mag. 41, 413 [1950]. 
Vermehrungen der Schauerteilchen stattfinden. Obwohl die Koinzidenzwahrscheinlichkeiten dann ausschließlich geometrisch bedingt waren, erwies sich die Rechnung doch noch als so kompliziert, daß statt dessen zu einer sogenannten ,Monte-Carlo-Metho-

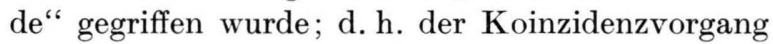
wurde in einer Modellanordnung so oft mit den aus Photoplattenbeobachtungen entnommenen statistischen Schwankungen nachgebildet, wie zur Erzielung ausreichender statistischer Genauigkeit erforderlich war.

Für eine Anzahl von Entstehungsorten in $S$ wurde jeweils eine Zentralprojektion der 3 Zählrohrlagen des Bleihauses auf eine gemeinsame Bezugsebene angefertigt und in dieser (mit elektrischen Kontakten) eine in der richtigen Weise schwankende Trefferstatistik, und damit die Ansprechwahrscheinlichkeit, ermittelt. Auf die Erzeugung der richtigen Trefferverteilung soll hier aus Raumgründen nicht näher eingegangen werden. Das nach Mittelung über 6 verschiedene Entstehungsorte erhaltene Ergebnis der Trefferstatistik ist in Abb. 10 wiedergegeben. Multiplikation mit der bei Photoplattenmessungen beobachteten Multiplizitätsverteilung ergibt die Kurven der Abb. 11, die somit die Multiplizitätsverteilungen der tatsächlich im Bleihaus registrierten Schauer darstellen, soweit die vereinfachenden Voraussetzungen zulässig waren.

Wie man sieht, tragen Schauer mit nur 3 geladenen Teilchen im Vergleich zu größeren Schauern nur sehr wenig zur Meßrate bei. Setzt man für den je Schauerteilchen im Mittel aufzuwendenden Energiebetrag 1,2-1,5 $\mathrm{GeV}$ an ${ }^{19}$, dann erhält man bei einer mittleren Multiplizität der registrierten Schauer von 5-7 Teilchen eine mittlere Schauerenergie 6--12 GeV; bei einer Verteilung, die von 3 bis etwa $20 \mathrm{GeV}$ reicht.

Die berechneten Koinzidenzraten (332, 432, 443, 554) verhalten sich nach Abb. 11 wie 100/40/25/3 zueinander, während die entsprechenden Messungen 100/70/25/10 ergaben. Die Übereinstimmung ist befriedigend, wenn man berücksichtigt, daß bei der Rechnung die Absorption und andere Sekundäreffekte vernachlässigt wurden und diese gerade bei den wenig energiereichen, strahlungsarmen Schauern von Bedeutung sind. Das gleiche gilt für einen Vergleich zwischen der berechneten und der gemessenen absoluten Koinzidenzzahl pro Minute.

C) Schauererzeugung in leichteren Elementen und in Wasserstoff

Bei der Materialabhängigkeit der Koinzidenzerzeugung fällt auf, daß die leichteren Elemente von Kohlenstoff bis Eisen bei gleichem Gesamtge- wicht auch etwa gleich viele Koinzidenzen erzeugen, obwohl die zugehörigen Kernstoßlängen sich erheblich voneinander unterscheiden $\left(\mathrm{Fe} \mathrm{100,} \mathrm{C} 60 \mathrm{~g} / \mathrm{cm}^{2}\right)$. Anscheinend findet also trotzdem immer die gleiche Anzahl an Primärstößen statt, wenn man nicht annehmen will, daß hier zufällig die Differenzen der Schauerproduktion gerade kompensiert werden durch entgegengesetzt auf die Meßrate einwirkende Unterschiede der sekundären Absorptions- und Streueffekte. Da das bei Materialdicken von nur einer geometrischen freien Weglänge jedoch sehr unwahrscheinlich ist, wird man vermuten dürfen,

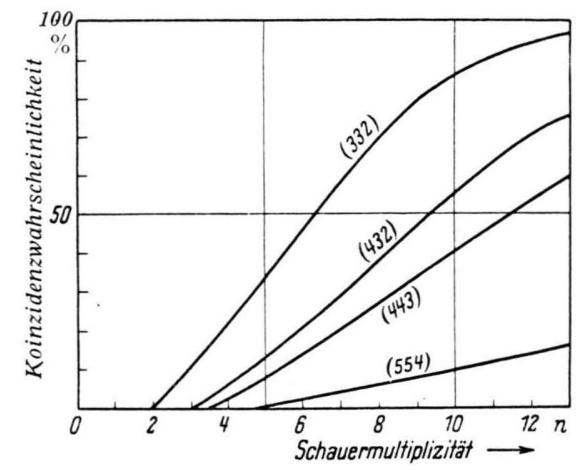

Abb. 10. Ansprechwahrscheinlichkeit der Koinzidenzapparatur gegenüber Schauern verschiedener Multiplizität. Wegen der Bestimmung der Kurven mittels einer ,Monte-Carlo-Methode“" s. S. 429, linke Spalte.

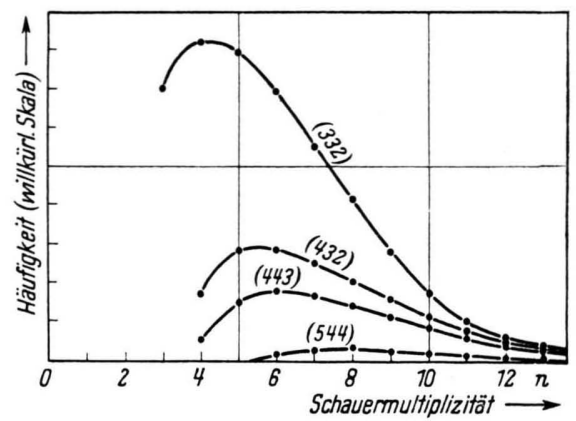

Abb. 11. Multiplizitätsverteilung der in der Apparatur registrierten harten Schauer. Die Kurven ergeben sich aus Abb. 10 und der natürlichen Entstehungswahrscheinlichkeit für Schauer bestimmter Multiplizitäten.

daß der auf das einzelne Nukleon bezogene Wirkungsquerschnitt bei der Schauererzeugung kleiner als der geometrische Kernquerschnitt ist, so daß die leichteren Atome noch als verhältnismäßig transparent anzusehen wären.

19 M. Teucher, Z. Naturforschg. 8a, 132 [1953]; J. Roederer, ebd. 7 a, 762 [1952]. 
$\mathrm{Zu}$ genaueren Abschätzungen der Abweichungen reicht die Meßgenauigkeit jedoch nicht aus, da beispielsweise die Differenz der Graphit- und Eisenkurven in Abb. 3 bei $50 \mathrm{~g} / \mathrm{cm}^{2}$ nur etwa das Doppelte der Fehlergrenzen betragen würde, wenn man als Maß für die Schichtdicke statt der Flächendichte $\left(\mathrm{g} / \mathrm{cm}^{2}\right)$ die Zahl der geometrischen freien Kernstoßlängen nähme. Sehr groß dürften die Abweichungen des wirksamen Nukleonenquerschnitts vom geometrischen Querschnitt wegen des beträchtlichen Intensitätsabfalls bei Blei anderseits auch nicht sein - es sei denn, der langsamere Anstieg der Meßrate mit wachsender Materialdicke würde in diesem Fall durch andere Multiplizitäts- und Richtungsverteilungen hervorgerufen, für deren Vorhandensein immerhin auch spricht, daß Auflockerungen des Materials $S$ die (332)-Koinzidenzrate bei Blei erheblich weniger beeinflussen als bei den leichteren Elementen.

Von besonderem Interesse sind die Vergleichsmessungen zwischen Paraffin und Graphit, aus denen hervorgeht, daß auch zwischen diesen beiden Materialien kein Unterschied in der Koinzidenzerzeugung besteht. Falls diese Übereinstimmung nur durch eine gleich große Schauerproduktion zustande käme, würde darin ein eindeutiger Beweis für die multiple $\pi$-Mesonenerzeugung zu erblicken sein, da nach den Darlegungen des vorhergehenden Abschnitts bei Kohlenstoff vorwiegend Schauer mit 4 oder mehr Teilchen gezählt werden, während solche Schauer in Wasserstoff nur nach der Theorie der Vielfacherzeugung, nicht aber nach der Theorie der $\pi$-Mesoneneinzelerzeugung entstehen können. Gegen diese Schlußweise lassen sich vor allem zwei Argumente anführen, die kurz besprochen seien.

Man könnte einwenden, die hohe Multiplizität bei den in Wasserstoff ausgelösten Schauern sei durch die beim Zerfall neutraler $\pi$-Mesonen entstehenden großen Elektronenkaskaden vorgetäuscht, da deren mittlere Energie bei Nukleon/Proton-StöBen wegen der fehlenden Sekundärstöße im Atomkern erheblich größer sei als bei den in schweren Atomkernen erzeugten Schauern. Mit den Energieverteilungen, die Camerini et al. für sekundäre Schauerteilchen in Photoplatten ${ }^{4,18}$ angeben, läßt sich jedoch abschätzen, daß die mittlere Energie für alle $\pi$-Mesonen zusammen bei Schauern mit 6 Mesonen, die in Kohlenstoffkernen entstanden sind, auch nach der Theorie der Einzelerzeugung mindestens noch halb so groß ist, wie die mittlere Energie der
Mesonen, die bei den ersten Zusammenstößen von Primärteilchen mit ruhenden Nukleonen erzeugt werden. Die mittlere Energie der Mesonen wäre dann zwar in Wasserstoff immerhin 1,2 GeV, so daß bis $\mathrm{zu}^{2} / 3$ von ihnen die Mindestenergie zur Erzeugung von Elektronenkaskaden hätten, die alle 3 Lagen des Bleihauses durchsetzen können. Wegen der Energieaufteilung auf zwei $\gamma$-Quanten, der Richtungsverteilung der Mesonen und der Bedingung, daß wenigstens ein Elektronenpaar schon oberhalb des Bleihauses ausgelöst werden muß, dürfte die so erzeugte (332)-Koinzidenzrate aber dennoch nur einen kleinen Bruchteil derjenigen ausmachen, die bei gleich viel Kohlenstoff von den entsprechend teilchenreicheren Schauern durch direkte Treffer harter Teilchen ausgelöst wird. Die viel häufigeren Kaskaden, die nur die beiden oberen Lagen durchsetzen, können andererseits Koinzidenzen nur dann auslösen, wenn gleichzeitig mindestens zwei geladene harte Teilchen die untere Zählrohrlage treffen. Wegen der breiten Winkelverteilung derselben dürfte das ebenfalls nur selten eintreten, abgesehen davon, daß dieser Fall bei $\pi$-Mesoneneinzelerzeugung nur mit primären Protonen möglich ist. Größere Beiträge zu den gemessenen Koinzidenzraten durch den Zerfall neutraler $\pi$-Mesonen sind deshalb auch beim Wasserstoff nicht zu befürchten.

Weiter könnte man einwenden, der beobachtete Wasserstoffanteil würde nicht durch eine entsprechende Schauerproduktion hervorgerufen, sondern durch eine Erhöhung der Zählwahrscheinlichkeit der aus dem Kohlenstoff des Paraffins kommenden Schauer, die den Ausfall der Schauererzeugung im Wasserstoff gerade kompensiert. Wegen der größeren Energieverzettelung bei sekundären Zusammenstößen mit Kohlenstoffatomen im Vergleich zu den Zusammenstößen mit Protonen dürfte ein solcher Effekt teilweise auch zweifellos bestehen, besonders, wenn es sich um die Erzeugung von Anstoßprotonen durch sekundäre Neutronen handelt. Eine sorgfältige Untersuchung aller in Frage kommenden Sekundärprozesse zeigt jedoch, daß die so entstehende mittlere Erhöhung der Strahlenzahl je Schauer höchstens $3 \%$ betragen kann. Aus der in Abb. 11 dargestellten Abhängigkeit der Meßrate von der Multiplizität läßt sich entnehmen, daß die Koinzidenzrate dadurch um etwa $6 \%$ zunehmen würde. Die tatsächlichen Werte dürften jedoch kleiner sein, da diese Abschätzungen der Maximalwerte unter der Voraussetzung geometrischer Wirkungsquerschnitte gemacht wurden, während nach den 
vorliegenden Messungen mit kleineren Stoßquerschnitten zu rechnen ist.

Es wird deshalb kaum zulässig sein, den gesamten bei $60 \mathrm{~g} / \mathrm{cm}^{2}$ Paraffin auf den Wasserstoff entfallenden Bruchteil $(10 \pm 2) \%$ der (332)-Koinzidenzrate nur auf solche Effekte zurückzuführen, wenn auch zugegeben werden muß, daß derartige Einflüsse hier nicht ohne weiteres vernachlässigt werden können. Das Ergebnis der Vergleichsmessungen zwischen Paraffin und Graphit dürfte daher mit großer Wahrscheinlichkeit für die Erzeugung teilchenreicher Schauer in Wasserstoff und damit für die multiple $\pi$-Mesonenerzeugung sprechen.

In letzter Zeit sind eine ganze Reihe anderer Arbeiten zur gleichen Fragestellung veröffentlicht worden ${ }^{13,15,20-23}$, in denen die Autoren teilweise $\mathrm{zu}$ entgegengesetzten Schlußfolgerungen kommen. Es sei noch kurz gezeigt, daß bei diesen Messungen innerhalb der Fehlergrenzen ein ernst zu nehmender Gegensatz zu den hier vorliegenden Ergebnissen dennoch nicht bestehen dürfte.

Die meisten Autoren gehen von der Voraussetzung aus, daß für die Erzeugung harter Schauer durchweg der geometrische Kernquerschnitt maßgeblich ist, während unsere Messungen nahelegen, daß statt dessen bei den leichten Elementen unabhängig von Atomgewicht Massenproportionalität besteht. Bei Berechnungen mit dem geometrischen Wirkungsquerschnitt würde man deshalb die Schauerproduktion in Wasserstoff z. B. gegenüber der in Aluminium um einen Faktor $\sqrt[3]{\mathbf{2 7}}=3$ überschätzen. Darin dürfte zu einem großen Teil die Erklärung für die im Sinne der multiplen $\pi$-Mesonenerzeugung negativen Resultate von Rolloson ${ }^{13}$, Walker et al. ${ }^{15}$ und Weaver ${ }^{21} \mathrm{zu}$ suchen sein. So hätte man beispielsweise im letzteren Fall bei Nebelkammerbeobachtungen unter flüssigem Wasserstoff innerhalb der Meßzeit statt der angegebenen 6 nur etwa 2-3 Schauer zu erwarten, abgesehen davon, daß die Abschätzung der geometrischen Unterschiede zwischen den Vergleichsmaterialien hier etwas opti-

${ }^{20}$ M. Vidale u. M. Schein, Nuovo Cim. 8, 774 [1951].

21 A. Weaver, Phys. Rev. 90, 86 [1953]. mistisch zu sein scheint. Der negative Ausfall des Experiments kann deshalb nicht mehr als ein Gegenbeweis gegen die Theorie der Vielfacherzeugung angesehen werden.

Sehr viel schwerwiegender dürften demgegenüber neuere Messungen von $\mathrm{Mc} \mathrm{Cusker}{ }^{23}$ sein, bei denen mit einer Apparatur, die der hier verwandten sehr ähnlich war, kein Wasserstoffanteil gefunden wurde. Bei Beschränkung auf die Schauer mit 4 oder mehr Teilchen - denn nur die können zum Vergleich mit den hier vorliegenden Messungen herangezogen werden - beträgt die Meßgenauigkeit jedoch nur noch etwa $8 \%$, während der auf Grund unserer Messungen zu erwartende Wasserstoffanteil an der Koinzidenzrate nur etwa $12 \%$ ausmachen sollte. Das negative Resultat dürfte sich deshalb auch in diesem Falle zwanglos als statistische Schwankung erklären lassen. Die Schlußweise von Mc Cusker wäre berechtigt, wenn die Schauerproduktion durch den geometrischen Wirkungsquerschnitt gegeben wäre, was aber nach unseren Messungen - wie schon betont - nicht der Fall zu sein scheint.

Auf die übrigen Arbeiten, für die ähnliche Überlegungen gelten, kann hier ebensowenig näher eingegangen werden, wie auf die vereinzelten Beobachtungen größerer Schauer in wasserstoffgefüllten Nebelkammern, gegen die sich wegen des Alkoholgehaltes Einwendungen erheben lassen, oder auf die zahlreichen Untersuchungen mit Photoplatten, die schon in der Einleitung erwähnt wurden. Abschließend läßt sich feststellen, da $B$ alle Untersuchungen anderer Autoren mit den hier gefundenen Ergebnissen durchaus verträglich sind und kein Anlaß besteht, auf Grund dieser Messungen die oben gegebene Erklärung der vorliegenden Meßergebnisse in $\mathbf{Z}$ weifel zu ziehen.

Diese Untersuchungen wurden in den Jahren 1949 bis 1952 am Max-Planck-Institut für Physik in Göttingen durchgeführt. Für die Anregung zu dieser Arbeit und das ständige Interesse an ihrem Fortgang möchte ich Herrn Professor $\mathrm{Haxel}$ besonders danken. Herrn Professor Heisenberg und Herrn Professor Wirtz bin ich für ihr stets förderndes Interesse und wertvolle Diskussionen ebenfalls zu großem Dank verpflichtet.

${ }^{22}$ C. Mc Cusker, H. Messel u. D. Millar, Phys. Rev. 89, 1172 [1953].

${ }^{23}$ C. Mc Cusker, N. Porter u. B. Wilson, Phys. Rev. 91, 384 [1953]. 